\title{
Tendências na prevalência de hipertensão arterial sistêmica e na utilização de serviços de saúde no Brasil ao longo de uma década (2008-2019)
}

\author{
Trends in the prevalence of systemic arterial hypertension \\ and health care service use in Brazil over a decade (2008-2019)
}

Nayara Abreu Julião (https://orcid.org/0000-0002-3223-9468) ${ }^{1}$

Aline de Souza (https://orcid.org/0000-0001-8860-9643) ${ }^{1}$

Raquel Rangel de Meireles Guimarães (https://orcid.org/0000-0003-1754-9238) ${ }^{2}$

${ }^{1}$ Centro de

Desenvolvimento e

Planejamento Regional, Universidade Federal de

Minas Gerais. Av. Presidente Antônio Carlos $6.6273^{\circ}$ andar, Pampulha. 31270-901 Belo Horizonte MG Brasil. najuliao@cedeplar.ufmg.br

${ }^{2}$ Departamento de

Economia, Universidade

Federal do Paraná. Curitiba

PR Brasil.

\begin{abstract}
The global burden and the prevalence of systemic arterial hypertension (SAH) have increased over the last two decades, especially in low- and middle-income countries, and are a concern to health authorities. This study analyzed the prevalence of SAH reported by Brazilian adults in 2008, 2013, and 2019, and individual disease control in 2013 and 2019. Data from the National Household Sample Survey (2008) and National Health Survey (2013-2019) were employed. We calculated the disease's prevalence ratios using Poisson regression, adjusted for sociodemographic characteristics. Regarding health care and PHC organization indicators, we calculated proportions by gender, age group, ethnicity, and region. The results reveal persistent regional inequalities, with lower prevalence in the North and Northeast and higher prevalence in the Southeast and South. While the health care access and use indicators are positive, reflecting PHC improvements in recent years, we highlight the importance of adopting multifaceted $S A H$ prevention and control strategies in the country.

Key words Systemic arterial hypertension, Prevalence, Health Care service use, Primary health care
\end{abstract}

Resumo A carga global e a prevalência de hipertensão arterial sistêmica (HAS) têm crescido nas últimas duas décadas, especialmente em países de baixa e média renda, representando uma preocupação para as autoridades em saúde. Este estudo analisou a prevalência de HAS entre adultos brasileiros em 2008, 2013 e 2019 e o controle da doença pelos indivíduos em 2013 e 2019. Utilizou-se dados da Pesquisa Nacional por Amostra de Domicílio (2008) e da Pesquisa Nacional de Saúde (2013-2019). Foram calculadas razões de prevalência da doença pelo método de Poisson, ajustado para caraterísticas sociodemográficas. Para os indicadores de cuidados em saúde e organização da atenção primária calculamos proporções estratificadas por sexo, faixa etária, raça e região. Os resultados indicam que as desigualdades regionais persistem, com menores prevalências no Norte e Nordeste e maiores no Sudeste e Sul. Embora os indicadores de acesso e utilização dos serviços de saúde sejam considerados bons, refletindo as melhorias na atenção primária nos últimos anos, ressaltamos a importância da adoção de estratégias multifacetadas para a prevenção e controle da HAS no país.

Palavras-chave Hipertensão arterial sistêmica, Prevalência, Utilização de serviços de saúde, Atenção Primária à Saúde 


\section{Introdução}

A Hipertensão Arterial Sistêmica (HAS), comumente conhecida como pressão arterial elevada, permanece sendo um dos principais determinantes de morbidade e mortalidade no país e no mundo. A HAS é tida como a principal causa de doenças cardiovasculares e de morte prematura $^{1}$. Embora relação de causalidade não esteja estabelecida, estudos apontam que pacientes hipertensos infectados pelo novo coronavírus (Sars-Cov-2) apresentam maiores chances de desenvolver desfechos clínicos desfavoráveis como a necessidade de cuidados intensivos e óbito ${ }^{2,3}$, o que representa um fator de risco para a população e preocupação para as autoridades em saúde com o aumento da exposição dos grupos de riscos e a consequente pressão sobre os sistemas de saúde.

Os custos econômicos das HAS podem ser medidos tanto de forma direta por meio dos gastos com internações e utilizações dos serviços de saúde, com a previdência social e com o absenteísmo, como de forma indireta através da perda da qualidade de vida medida pelo DALY (anos de vida ajustado por incapacidade) e da perda de produtividade. Ao considerar as internações hospitalares, os atendimentos ambulatoriais e os gastos com medicamentos em 2018, Nilson et $\mathrm{al}^{4}{ }^{4}$ estimaram os custos atribuíveis à HAS para o Sistema Único de Saúde (SUS) na ordem de mais de R $\$ 2$ bilhões por ano. Segundo um estudo realizado na cidade de São Paulo, a HAS está entre as principais doenças que, se eliminadas, trariam maior benefícios para a população idosa em termos de expectativa de vida livre de incapacidade ${ }^{5}$.

No Brasil são mais de 38 milhões de brasileiros, com 18 anos ou mais, diagnosticados com a doença, de acordo com a Pesquisa Nacional de Saúde (2019). Embora haja uma tendência global ascendente das taxas de HAS, esse aumento não ocorre de forma uniforme entre as economias. Países de média e baixa renda têm apresentado um crescimento mais expressivo $(31,5 \%)$ do que os países de renda alta $(28,5 \%)^{6}$. O aumento na prevalência de HAS reflete diferentes aspectos da condição de vida da população. O envelhecimento populacional, somado à adoção de estilos de vida não saudáveis, com a priorização de alimentos ultra processados, consumo de álcool, tabagismo e falta de atividades físicas, têm contribuído para esta elevação. Outros aspectos como o conhecimento, o controle e o tratamento da HAS também são altamente sensíveis aos atributos individuais e socioeconômicos ${ }^{7}$.
A prevenção, o controle e o tratamento da HAS exigem ações integradas e coordenadas entre o sistema de saúde, profissionais da saúde, indivíduos e comunidade. Do ponto de vista da organização do sistema e da oferta de serviços, a expansão da Estratégia Saúde Família (ESF) a partir dos anos 2000 representou um passo importante na redução das iniquidades em saúde e na ampliação do acesso aos serviços básicos de saúde na atenção primária. Os resultados positivos da implementação da ESF podem ser demonstrados pelas reduções da mortalidade e das hospitalizações por condições sensíveis à atenção primária ${ }^{8}$. Programas especiais visando o controle das duas condições mais prevalentes na população brasileira também foram implementados nessa década. O Hiperdia, direcionado aos usuários do SUS com Hipertensão Arterial Sistêmica e Diabetes Mellitus, realiza o cadastro e acompanhamento desses usuários e distribui os medicamentos necessários ao tratamento na rede básica de saúde ${ }^{9,10}$. O Programa Farmácia Popular do Brasil (PFPB), criado em 2004, no âmbito da política de assistência farmacêutica, já beneficiou mais de um terço dos hipertensos e diabéticos, especialmente as camadas mais vulneráveis, por meio da obtenção gratuita de pelo menos um medicamento ${ }^{11}$.

A participação dos usuários no controle da doença também é parte fundamental do processo. O uso contínuo e correto da medicação somado à adoção de um estilo de vida saudável reduz as chances de complicações pela doença, resultando em menor sobrecarga dos sistemas de saúde e maior qualidade de vida para os indivíduos. Todavia, a baixa adesão aos medicamentos é tida como a principal falha no controle da $\mathrm{HAS}^{12}$. O caráter silencioso e muitas vezes assintomático desafia o diagnóstico precoce e o tratamento da HAS. Não é incomum o relato de usuários que deixam de buscar assistência médica ou descontinuam o tratamento devido à ausência de sintomas ou à normalização dos níveis pressóricos ${ }^{13}$. Outros fatores relacionados à baixa adesão às medidas medicamentosas são os fatores sociodemográficos como idade; nível de escolaridade e renda; compreensão e percepção dos riscos da doença pelo usuário; vínculos estabelecidos entre os profissionais de saúde e os usuários; e questões relacionadas à organização dos sistemas de saúde ${ }^{12-14}$.

Diante desse contexto, este estudo tem dois objetivos. O primeiro é analisar a tendência de prevalência de HAS para a população brasileira com 18 anos ou mais, nos anos de 2008-2013- 
2019. Para esta análise, utilizamos dados extraídos da Pesquisa Nacional por Amostra de Domicílios (PNAD) e da Pesquisa Nacional de Saúde (PNS, 2013-2019). O segundo objetivo é avaliar os indicadores de cuidados em saúde pelos pacientes hipertensos. Essa parte da análise se restringe aos anos de 2013 e 2019, uma vez que este tema foi coberto apenas pela PNS. Estudo similar analisando este módulo específico da pesquisa para o ano de 2013 já foi desenvolvido por Malta et al. ${ }^{15}$ Ampliamos o escopo de análise incorporando as estatísticas mais recentes com o intuito de monitorar a evolução do cuidado. Conhecer a distribuição da doença no território nacional, bem como questões relacionadas ao acesso, cuidado e tratamento da condição é de fundamental importância para o delineamento de estratégias em saúde que visem ações de prevenção e controle da HAS no país, abordando questões relativas ao nível do indivíduo, do sistema de saúde e da comunidade.

\section{Metodologia}

\section{Desenho e população de estudo}

Para analisar a tendência na prevalência da HAS e os indicadores de cuidados em saúde pela população hipertensa acima de 18 anos utilizamos três inquéritos populacionais de abrangência nacional realizados pelo Instituto Brasileiro de Geografia e Estatística (IBGE), nos anos de 2008, 2013 e 2019. A PNAD, referente ao ano de 2008, abordou em seu questionário suplementar aspectos relacionados à saúde, acesso e utilização dos serviços de saúde pelos brasileiros. Tendo em vista a necessidade de mais informações sobre os indicadores de saúde e o funcionamento do sistema de saúde no país, o Ministério da Saúde, em parceria com o IBGE e a Fundação Oswaldo Cruz (Fiocruz), elaborou uma pesquisa específica para este fim. A PNS, projetada para ter periodicidade quinquenal, foi a campo pela primeira vez em 2013 e em 2019 teve sua segunda edição, permitindo ampliar o escopo sobre questões relacionadas à saúde em território nacional.

A PNAD adota um plano amostral estratificado com um, dois ou três estágios de seleção dependendo do estrato ${ }^{16}$. Por sua vez, a PNS adota o desenho amostral da Amostra Mestra de pesquisas domiciliares do IBGE, com amostragem probabilística em três estágios. Primeiro é realizado a estratificação das unidades primárias de amostragem (UPA). Em seguida, os domicílios são selecionados dentro de cada setor censitário a partir do Cadastro Nacional de Endereços para Fins Estatísticos (CNEFE) por amostragem aleatória simples (AAS). Por último um morador é selecionado dentro de cada domicílio por AAS para responder a blocos temáticos específicos. A idade mínima do morador selecionado diferiu entre as duas edições da pesquisa, sendo de 18 anos em 2013 e de 15 anos em $2019^{17}$.

O questionário da PNS é dividido em três partes. A primeira aborda questões sobre o domicílio e visitas dos agentes de endemias e são respondidas pelo responsável pelo domicílio. A segunda contém informações gerais relativas a todos os moradores do domicílio, em que um morador com 18 anos ou mais responde por todos os demais. Essa primeira parte da pesquisa dá continuidade aos Suplementos de Saúde da PNAD. Na terceira parte são investigados temas específicos como características de trabalho e de apoio social, percepção do estado de saúde, acidentes e violências, hábitos de saúde e estilo de vida, doenças crônicas, saúde da mulher, atendimento pré-natal, saúde bucal e atendimento médico, sendo respondidas somente pelo morador selecionado $^{17,18}$.

Sendo assim, o presente estudo está dividido em duas etapas metodológicas. Na primeira, descrevemos a tendência na prevalência de HAS autorreferida na população brasileira entre 2008 e 2019, segundo características sociodemográficas. Na segunda etapa, descrevemos os cuidados em saúde pela população que reportou a presença da condição em 2013 e 2019 (conforme o módulo Q da Pesquisa Nacional de Saúde). Embora o questionário tenha passado por algumas revisões entre uma edição e outra, a maior parte do questionário manteve-se compatível com a primeira edição ${ }^{17}$.

Para garantir a comparabilidade dos resultados ao longo dos anos, foram considerados apenas os indivíduos com 18 anos ou mais. Dos 391.868 indivíduos investigados na PNAD, foram selecionados 271.677 indivíduos. Na PNS, em 2013, foram selecionados 60.202 indivíduos com 18 anos ou mais e, em 2019, dos 90.846 moradores selecionados com 15 anos ou mais, permaneceram na amostra 88.531 indivíduos.

\section{Variáveis de estudo}

A condição de HAS autorreferida foi obtida através da seguinte pergunta: Algum médico já lhe disse que o(a) Sr.(a) tem hipertensão arterial (pressão alta)? sendo considerado hipertensos os indivíduos que responderam sim à questão; 
Características sociodemográficas: sexo; faixa etária (18-29 anos; 30-59 anos; 60-64 anos; 65-74 anos; 75 anos ou mais), raça/cor (branca, preta e parda); nível de escolaridade (sem instrução ou Fundamental incompleto, Fundamental completo ou Médio incompleto, Médio completo ou Superior incompleto, Superior completo); região geográfica; área de residência (urbano ou rural). As categorias "Amarela" e "Indígenas" foram agrupadas como "outras" e consideradas na amostra no cálculo de proporção, mas em função da baixa frequência foi omitida nos resultados. $\mathrm{Na}$ análise de regressão de Poisson para o cálculo das razões de prevalência esta categoria foi excluída da análise;

Variáveis de cuidados em saúde entre os hipertensos: percentual de indivíduos que tomaram medicamentos para HAS nas duas últimas semanas; percentual de indivíduos que receberam assistência para HAS nos últimos doze meses; percentual de indivíduos que realizaram o último atendimento nas Unidades Básicas de Saúde e percentual de indivíduos que realizaram o último atendimento em consultórios particulares; percentual de indivíduos que realizaram atendimentos pelo Sistema Único de Saúde (SUS); percentual de indivíduos que teve exame solicitados e que conseguiu realizara todos os exames solicitados; percentual que tiveram alguma pactuação de plano de cuidado junto aos profissionais de saúde (inclui, por exemplo, a manutenção de alimentação saudável, prática regular de atividade física, diminuição do consumo de sal, entre outras.); percentual de indivíduos que realizou todas as consultas com o mesmo médico; percentual de indivíduos que teve todas as consultas com médico especialista ou foi encaminhado e conseguiu realizar todos as consultas com médico especialista; percentual de indivíduos que esteve internado por causa da HAS ou alguma outra complicação; percentual de indivíduos que tem suas atividades habituais limitadas de forma intensa ou muito intensa devido à HAS; A variável foi construída da seguinte maneira: se o indivíduo respondeu "sim" a pelo menos uma das perguntas de Q01801 a Q01808 em 2013 e Q018010-Q018017 em 2019.

Variável de cobertura da ESF: percentual de moradores em domicílios cadastrado na Estratégia Saúde da Família.

\section{Métodos de análise}

As análises foram conduzidas utilizando o software estatístico Stata versão 14.0. Para a precisão correta dos estimadores foi utilizado o pacote para análise de dados provenientes de amostra complexa (survey) que incorpora informações do plano amostral: unidade primária de amostragem (UPA), estrato e pesos amostrais. Inicialmente, são apresentadas as prevalências de HAS na população brasileira adulta, acima dos 18 anos, para os anos de 2008, 2013 e 2019. Foram calculadas as razões de prevalência de HAS para os três anos através do método de Poisson, ajustadas por sexo, raça, faixa etária, nível de escolaridade, região e área de residência. O método de Poisson para o cálculo de prevalência tem sido utilizado por outros estudos que apontam para o melhor ajuste do modelo para desfechos frequentes $^{19,20}$. Em seguida, para a amostra de indivíduos que referiram a condição em 2013 e 2019, são apresentadas as proporções relativas aos indicadores de cuidados em saúde e cobertura da ESF estratificadas por sexo, faixa etária, raça e região. Utilizamos o teste qui-quadrado de Pearson ajustado para comparações entre os anos.

\section{Resultados}

\section{Prevalência de hipertensão arterial sistêmica na população brasileira}

Na Tabela 1 são apresentados os resultados de prevalência de HAS para os adultos brasileiros e as razões de prevalência de HAS ajustadas por características sociodemográficas. Em 2008, 19,9\% ( IC $\left._{95}: 19,7-20,1\right)$ reportaram o diagnóstico de HAS, representando mais de 26 milhões de adultos brasileiros com 18 anos ou mais, considerando a expansão da amostra. Dos 60.202 indivíduos em 2013, 3,0\% ( $\mathrm{IC}_{95}:$ 2,7-3,2) nunca tiveram pressão arterial aferida e $21,3 \%\left(\mathrm{IC}_{95}\right.$ : 20,8-22,0) reportaram o diagnóstico de HAS, 0 que representa mais de 31 milhões de brasileiros. Em 2019, 2,0\% ( IC $\left._{95}: 1,8-2,2\right)$ nunca tiveram pressão arterial aferida e 23,9\% ( IC $\left._{95}: 23,4-24,4\right)$ tinham diagnóstico da condição, cerca de 38,1 milhões de adultos brasileiros.

Nos três períodos analisados, observa-se maior prevalência de HAS entre as mulheres, com crescimento entre as faixas etárias. Maiores prevalências são encontradas entre os indivíduos que se autodeclararam pretos, seguidos dos brancos e pardos. Com relação ao nível de escolaridade, a prevalência da condição é maior entre os indivíduos com menor nível de escolaridade. Entre os indivíduos sem instrução ou com Ensino Fundamental incompleto, $28,7 \%\left(\mathrm{IC}_{95}: 28,3-\right.$ 
Tabela 1. Prevalência (\%) de hipertensão arterial sistêmica referida na população com 18 anos ou mais e Razão de Prevalência (RP) de hipertensão ajustada por características sociodemográficas e regionais.

\begin{tabular}{|c|c|c|c|c|c|c|}
\hline \multirow{2}{*}{ Variáveis } & \multicolumn{2}{|r|}{2008} & \multicolumn{2}{|r|}{2013} & \multicolumn{2}{|r|}{2019} \\
\hline & $\%$ & $\mathbf{R P}\left(\mathrm{IC}_{95}\right)$ & $\%$ & $\mathbf{R P}\left(\mathrm{IC}_{95}\right)$ & $\%$ & $\mathbf{R P}\left(\mathrm{IC}_{95}\right)$ \\
\hline Nunca teve PA aferida & - & & 3,0 & & 2,0 & \\
\hline Hipertensão referida & 19,9 & & 21,4 & & 23,9 & \\
\hline \multicolumn{7}{|l|}{ Sexo } \\
\hline Masculino & 16,4 & 1 & 18,1 & 1 & 21,1 & 1 \\
\hline Feminino & 23,0 & $1,3(1,3-1,3)$ & 24,3 & $1,3(1,2-1,3)$ & 26,4 & $1,2(1,1-1,2)$ \\
\hline \multicolumn{7}{|l|}{ Raça } \\
\hline Branca & 20,2 & 1 & 22,1 & 1 & 24,4 & 1 \\
\hline Preta & 24,7 & $1,3(1,2-1,3)$ & 24,2 & $1,1(1,0-1,2)$ & 25,8 & $1,2(1,1-1,2)$ \\
\hline Parda & 18,6 & $1,0(1,0-1,1)$ & 20,0 & $1,0(1,0-1,1)$ & 22,9 & $1,1(1,0-1,1)$ \\
\hline \multicolumn{7}{|l|}{ Faixa etária } \\
\hline $18-29$ & 2,9 & 1 & 2,9 & 1 & 2,8 & 1 \\
\hline $30-59$ & 19,5 & $6,3(6,0-6,6)$ & 20,5 & $6,5(5,6-7,5)$ & 20,3 & $6,5(5,6-7,6)$ \\
\hline $60-64$ & 48,4 & $14,3(13,5-15,0)$ & 44,2 & $12,9(11,0-15,2)$ & 46,9 & $13,8(11,8-16,3)$ \\
\hline $65-74$ & 54,4 & $15,7(14,9-16,5)$ & 53,0 & $15,0(12,8-17,6)$ & 56,6 & $16,3(14,0-19,1)$ \\
\hline 75 ou mais & 57,1 & $16,0(15,2-16,8)$ & 55,0 & $15,2(12,9-17,8)$ & 62,1 & $17,3(14,8-20,3)$ \\
\hline \multicolumn{7}{|l|}{ Escolaridade } \\
\hline $\begin{array}{l}\text { Sem instrução e Ensino } \\
\text { Fundamental incompleto }\end{array}$ & 28,7 & $1,5(1,4-1,6)$ & 31,1 & $1,4(1,3-1,5)$ & 36,6 & $1,5(1,4-1,6)$ \\
\hline $\begin{array}{l}\text { Ensino Fundamental } \\
\text { completo e Médio } \\
\text { incompleto }\end{array}$ & 14,2 & $1,2(1,2-1,3)$ & 16,7 & $1,2(1,1-1,4)$ & 20,4 & $1,3(1,2-1,5)$ \\
\hline $\begin{array}{l}\text { Ensino Médio completo e } \\
\text { Superior incompleto }\end{array}$ & 10,6 & $1,0(1,0-1,0)$ & 13,4 & $1,0(0,9-1,1)$ & 15,4 & $1,1(1,0-1,2)$ \\
\hline Ensino Superior completo & 15,4 & 1 & 17,9 & 1 & 18,2 & 1 \\
\hline \multicolumn{7}{|l|}{ Área de residência } \\
\hline Rural & 18,9 & 1 & 19,8 & 1 & 23,2 & 1 \\
\hline Urbano & 20,1 & $1,1(1,1-1,2)$ & 21,6 & $1,1(1,1-1,2)$ & 24,0 & $1,1(1,1-1,2)$ \\
\hline \multicolumn{7}{|l|}{ Região } \\
\hline Norte & 14,1 & 1 & 14,4 & 1 & 16,8 & 1 \\
\hline Nordeste & 18,2 & $1,1(1,1-1,2)$ & 19,5 & $1,2(1,1-1,3)$ & 23,1 & $1,2(1,2-1,3)$ \\
\hline Sudeste & 21,7 & $1,3(1,2-1,4)$ & 23,3 & $1,3(1,2-1,5)$ & 25,9 & $1,4(1,3-1,5)$ \\
\hline Sul & 21,0 & $1,3(1,2-1,4)$ & 22,8 & $1,4(1,3-1,5)$ & 24,5 & $1,3(1,2-1,4)$ \\
\hline Centro-Oeste & 18,6 & $1,2(1,2-1,3)$ & 21,1 & $1,3(1,2-1,4)$ & 21,9 & $1,3(1,2-1,3)$ \\
\hline $\mathrm{N}$ & 271.667 & 267.965 & 60.202 & 57.498 & 88.531 & 85.553 \\
\hline
\end{tabular}

Fonte: PNAD (2008) e PNS (2013 e 2019).

29,0) referiram o diagnóstico de HAS em 2008, chegando a 32,4\% ( $\left.\mathrm{IC}_{95}: 31,2-33,6\right)$ em 2013, e $37,8 \%\left(\mathrm{IC}_{95}: 36,9-38,7\right)$ em 2019 . As distribuições por região geográfica e área de residência apontam para a maior prevalência nas regiões Sudeste e Sul e nas áreas urbanas.

As razões de prevalência (RP) apresentadas nas colunas da Tabela 1 demonstram que, mesmo após ajustados por características sociodemográficas, as mulheres têm de duas a três vezes mais chances de reportarem diagnóstico de HAS em 
de escolaridade têm maiores chances de reportar HAS em comparação àqueles com ensino superior completo, exceto em 2013 para a categoria Ensino Médio completo e Superior incompleto, em que a maior chance não é estatisticamente significante. Em relação à região Norte, os moradores de todas as demais regiões geográficas têm maiores chances de reportar diagnóstico de HAS, revelando um padrão de desigualdades regionais persistente.

\section{Cuidado em saúde pela população com diagnóstico de hipertensão arterial sistêmica}

A segunda parte da análise é direcionada aos indivíduos que alegaram ter diagnóstico de Hipertensão Arterial Sistêmica em 2013 e 2019. A Tabela 2 apresenta os indicadores de cuidados em saúde segundo o sexo. A proporção dos indivíduos que tomaram medicamentos para HAS nas duas últimas semanas foi de $81,6 \%$ (77,1\% dos homens e $84,6 \%$ das mulheres) em 2013 e de $88 \%$ (84,2\% dos homens e $90,7 \%$ das mulheres) em 2019. Receberam assistência médica $69,7 \%$ dos indivíduos em 2013 (65,6\% dos homens e 72,5\% das mulheres) e $72,2 \%(68,9 \%$ dos homens e $74,5 \%$ das mulheres) em 2019. Nos dois anos, cerca de $65,6 \%$ (61\% dos homens e $68 \%$ das mulheres) receberam atendimento pelo SUS. A Unidade Básica de Saúde foi o local com maior proporção de atendimentos na última assistência, aproximadamente $46 \%$ para os dois anos. Em seguida, o local com mais atendimentos foi o consultório particular. Enquanto a proporção de mulheres atendidas na UBS foi maior que a de homens nos dois períodos, nos consultórios particulares essa relação se inverte sendo a proporção de homens atendidos maior que a de mulheres. Em 2013, $56,5 \%$ (54,9\% dos homens e $57,4 \%$ das mulheres) realizaram a última consulta com o mesmo médico das consultas anteriores, enquanto em 2019 a proporção foi similar para ambos os sexos, aproximadamente $52 \%$. Cerca de $92 \%$ dos indivíduos em 2013 e 90\% em 2019 realizaram todos os exames solicitados, sendo similar a proporção entre os sexos em 2019. Mais de 95\% de homens e mulheres receberam pelo menos uma recomendação dos profissionais de saúde relacionada ao plano de cuidado nos dois anos. A proporção dos que fizeram consultas com especialistas também

Tabela 2. Indicadores de cuidados em saúde pelos indivíduos com hipertensão arterial sistêmica segundo sexo.

\begin{tabular}{|c|c|c|c|c|c|c|}
\hline \multirow[b]{2}{*}{ Variáveis } & \multicolumn{3}{|c|}{2013} & \multicolumn{3}{|c|}{2019} \\
\hline & $\begin{array}{c}\text { Total } \\
(\%)\end{array}$ & $\begin{array}{l}\text { Homens } \\
(\%)\end{array}$ & $\begin{array}{l}\text { Mulheres } \\
(\%)\end{array}$ & $\begin{array}{c}\text { Total } \\
(\%)\end{array}$ & $\begin{array}{l}\text { Homens } \\
(\%)\end{array}$ & $\begin{array}{c}\text { Mulheres } \\
(\%)\end{array}$ \\
\hline $\begin{array}{l}\text { Tomou medicamentos para hipertensão nas } \\
\text { últimas duas semanas }\end{array}$ & 81,6 & 77,1 & 84,6 & 88,0 & 84,2 & 90,7 \\
\hline $\begin{array}{l}\text { Recebeu assistência nos últimos } 12 \text { meses para } \\
\text { hipertensão }\end{array}$ & 69,7 & 65,6 & 72,5 & 72,2 & 68,9 & 74,5 \\
\hline \multicolumn{7}{|l|}{ Local do atendimento } \\
\hline Unidade Básica de Saúde & 46,0 & 40,9 & 49,2 & 45,8 & 41,8 & 48,5 \\
\hline Consultório particular & 24,5 & 27,7 & 22,5 & 28,8 & 32,3 & 26,5 \\
\hline Atendimento pelo SUS & 65,6 & 61,0 & 68,5 & 65,6 & 61,3 & 68,5 \\
\hline $\begin{array}{l}\text { Última consulta com mesmo médico das consultas } \\
\text { anteriores }\end{array}$ & 56,5 & 54,9 & 57,4 & 51,9 & 52,3 & 51,7 \\
\hline Fez todos os exames solicitados & 91,9 & 92,2 & 91,8 & 89,6 & 90,5 & 89,0 \\
\hline $\begin{array}{l}\text { Pactuação de plano de cuidados com profissionais } \\
\text { da saúde }\end{array}$ & 96,5 & 96,7 & 96,4 & 95,1 & 95,2 & 95,0 \\
\hline $\begin{array}{l}\text { Fez todas as consultas ou foi encaminhado e } \\
\text { conseguiu fazer consultas com especialistas }\end{array}$ & 87,3 & 86,9 & 87,6 & 90,4 & 90,5 & 90,2 \\
\hline $\begin{array}{l}\text { Alguma vez esteve internado por causa da } \\
\text { hipertensão ou complicação }\end{array}$ & 14,0 & 12,5 & 14,9 & 12,7 & 11,2 & 13,7 \\
\hline $\begin{array}{l}\text { Tem suas atividades habituais limitadas devido à } \\
\text { hipertensão de forma intensa ou muito intensa. }\end{array}$ & 4,7 & 4,2 & 5,0 & 3,0 & 3,1 & 3,0 \\
\hline
\end{tabular}

Nota: Valores em negrito não foram estatisticamente significantes na comparação entre os anos pelo teste qui quadrado de Pearson ajustado, ao nível de $5 \%$. 
foi próxima entre os dois grupos, aproximadamente $87 \%$ em 2013 e 90\% em 2019. Já a proporção de indivíduos que tiveram internação foi de $14 \%$ (12,5\% dos homens e $14,9 \%$ das mulheres) em 2013 e $12,7 \%$ (11,2\% dos homens e $13,7 \%$ das mulheres) em 2019. As atividades habituais foram limitadas de forma intensa ou muito intensa para $4,7 \%$ (4,2\% dos homens e $5 \%$ das mulheres) dos indivíduos em 2013 e cerca de 3\% para ambos os sexos em 2019.

Em relação aos indicadores de cuidados em saúde segundo cor ou raça autodeclarada, 84,7\% dos brancos e menos de $80 \%$ de pretos e pardos tomaram medicamentos em 2013, enquanto em 2019 as proporções foram de $89,4 \%$ para brancos, $88,0 \%$ para pretos e $86,5 \%$ para pardos. Em 2013, a proporção dos que receberam assistência médica foi próxima para os três grupos, cerca de 70\%, e em 2019 foi aproximadamente de $72 \%$ entre brancos e pardos e $74,7 \%$ entre pretos. $\mathrm{O}$ atendimento pelo SUS nos dois anos foi superior a $70 \%$ entre pretos e pardos e entre brancos não chegou a $60 \%$ no período. Pretos e pardos apresentaram maior proporção de atendimentos na UBS do que brancos nos dois anos. $\mathrm{O}$ atendimento em consultório particular entre brancos foi de $31,8 \%$ em 2013 e $38,6 \%$ em 2019 , já entre pretos e pardos não chegou a $20 \%$ em 2013 e ficou em torno de $20 \%$ em 2019. A última consulta foi realizada com o mesmo médico das consultas anteriores para $62,3 \%$ dos brancos e cerca de $51 \%$ dos pretos e pardos em 2013 e para $56,7 \%$ dos brancos e menos de $49 \%$ dos pretos e pardos em 2019. Nos dois períodos mais de $90 \%$ dos brancos fizeram todos os exames solicitados, enquanto entre pretos e pardos as proporções foram de $85,9 \%$ e $90,7 \%$ em 2013 e $87 \%$ e $88 \%$ em 2019 , respectivamente. Para os três grupos, mais de $96 \%$ em 2013 e cerca de 95\% em 2019 receberam recomendações dos profissionais de saúde em relação ao plano de cuidados. Consultas com especialistas foram realizadas por mais de $90 \%$ dos brancos nos dois anos, por 80,9\% dos pretos e $84,6 \%$ dos pardos em 2013 e cerca de $88 \%$ de pretos e pardos em 2019. Estiveram internados devido à HAS 13,3\% dos brancos e aproximadamente $15 \%$ dos pretos e pardos em 2013 e $11 \%$ dos brancos e cerca de $14 \%$ de pretos e pardos em 2019. Em 2013, cerca de $4 \%$ de brancos e pretos e $5 \%$ de pardos responderam que a HAS limita as atividades habituais de forma intensa ou muito intensa, enquanto em 2019 essa limitação ocorreu para 2,2\% dos brancos e quase $4 \%$ dos pretos e pardos (Tabela 3 ).
Considerando os indicadores de cuidados em saúde segundo a faixa etária, observa-se um aumento na proporção de indivíduos que tomaram medicamentos para HAS nos dois anos, chegando a $92,3 \%$ e $96,7 \%$ para aqueles com 75 anos ou mais em 2013 e 2019, respectivamente. Em 2013, $60,6 \%$ dos indivíduos entre 18 e 29 anos, 68,3\% entre 30 e 59 anos e mais de $70 \%$ entre os com 60 anos ou mais receberam assistência médica, enquanto em 2019 as proporções foram de 62,9\% entre os de 18 a 29 anos e acima de $70 \%$ entre os de 30 anos ou mais. A proporção de indivíduos atendidos na UBS aumentou com a progressão da idade nos dois anos, exceto para o grupo de 75 anos ou mais, que apresentou proporção inferior à das faixas etárias de 30 a 74 anos. Já o atendimento em consultório particular foi maior entre os com 75 anos ou mais. Para o indicador de última consulta realizada com o mesmo médico das consultas anteriores, observa-se um crescimento entre as faixas etárias, sendo a proporção acima de $60 \%$ em 2013 e acima de 55\% em 2019 para os grupos com 65 anos ou mais. Nos dois anos, a proporção de indivíduos com 60 anos ou mais que realizou todos os exames solicitados e que fez consulta com especialistas foi superior a $90 \%$. Embora as recomendações dos profissionais de saúde em relação ao plano de cuidados tenham proporções altas entre todas as faixas etárias nos dois anos, este indicador foi maior para o grupo de 60 a 64 anos. A proporção dos que tiveram internação ou que relataram que a HAS limita intensamente ou muito intensamente a realização das atividades habituais foi menor entre o grupo de 18 a 29 anos nos dois anos e maior entre o grupo com 75 anos ou mais (Tabela 4).

A Tabela 5 apresenta a distribuição de indicadores de cuidados em saúde e da cobertura da ESF segundo as grandes regiões do país. Destacamos a proporção de indivíduos que fizeram consulta nos últimos 12 meses menor no Centro-Oeste, não chegando a $70 \%$ em nenhum dos dois anos. O Sudeste foi a região com menor proporção de indivíduos que tiveram internação devido a complicações da HAS nos dois anos, enquanto o Nordeste foi a região com maior proporção de internações em 2013 e o Centro-Oeste em 2019. Nos dois períodos, o atendimento pelo SUS foi mais frequente entre moradores do Norte e Nordeste, enquanto o Sudeste apresentou as menores proporções tanto em 2013 quanto em 2019. A cobertura populacional da ESF foi maior no Nordeste e Sul e menor no Sudeste, nos dois anos. 
Tabela 3. Indicadores de cuidados em saúde pelos indivíduos com hipertensão arterial sistêmica segundo cor/ raça.

\begin{tabular}{|c|c|c|c|c|c|c|c|c|}
\hline \multirow[b]{2}{*}{ Variáveis } & \multicolumn{4}{|c|}{2013} & \multicolumn{4}{|c|}{2019} \\
\hline & $\begin{array}{c}\text { Total } \\
(\%)\end{array}$ & $\begin{array}{c}\text { Branca } \\
(\%)\end{array}$ & $\begin{array}{c}\text { Preta } \\
(\%)\end{array}$ & $\begin{array}{c}\text { Parda } \\
(\%)\end{array}$ & $\begin{array}{l}\text { Total } \\
(\%)\end{array}$ & $\begin{array}{c}\text { Branca } \\
(\%)\end{array}$ & $\begin{array}{c}\text { Preta } \\
(\%)\end{array}$ & $\begin{array}{c}\text { Parda } \\
(\%)\end{array}$ \\
\hline $\begin{array}{l}\text { Tomou medicamentos para hipertensão nas } \\
\text { últimas duas semanas }\end{array}$ & 81,6 & 84,7 & 79,6 & 78,5 & 88,0 & 89,4 & 88,0 & 86,5 \\
\hline $\begin{array}{l}\text { Recebeu assistência nos últimos } 12 \text { meses } \\
\text { para hipertensão }\end{array}$ & 69,7 & 70,1 & 70,4 & 69,4 & 72,2 & 71,5 & 74,7 & 72,1 \\
\hline \multicolumn{9}{|l|}{ Local do atendimento } \\
\hline Unidade Básica de Saúde & 46,0 & 41,4 & 50,0 & 50,9 & 45,8 & 39,4 & 51,9 & 50,7 \\
\hline Consultório particular & 24,5 & 31,8 & 16,4 & 17,3 & 28,8 & 38,6 & 20,0 & 21,3 \\
\hline Atendimento pelo SUS & 65,6 & 57,0 & 77,8 & 73,4 & 65,6 & 55,3 & 74,6 & 73,6 \\
\hline $\begin{array}{l}\text { Última consulta com mesmo médico das } \\
\text { consultas anteriores }\end{array}$ & 56,5 & 62,3 & 50,8 & 50,5 & 51,9 & 56,7 & 48,8 & 48,2 \\
\hline Fez todos os exames solicitados & 91,9 & 94,1 & 85,9 & 90,7 & 89,6 & 91,6 & 87,0 & 88,0 \\
\hline $\begin{array}{l}\text { Pactuação de plano de cuidados com } \\
\text { profissionais da saúde }\end{array}$ & 96,5 & 96,7 & 96,6 & 96,3 & 95,1 & 95,4 & 95,2 & 94,6 \\
\hline $\begin{array}{l}\text { Fez todas as consultas ou foi encaminhado e } \\
\text { conseguiu fazer consultas com especialistas }\end{array}$ & 87,3 & 90,8 & 80,9 & 84,6 & 90,4 & 92,8 & 87,9 & 88,3 \\
\hline $\begin{array}{l}\text { Alguma vez esteve internado por causa da } \\
\text { hipertensão ou complicação }\end{array}$ & 14,0 & 13,3 & 15,2 & 14,5 & 12,7 & 11,0 & 13,6 & 14,2 \\
\hline $\begin{array}{l}\text { Tem suas atividades habituais limitadas } \\
\text { devido à hipertensão de forma intensa ou } \\
\text { muito intensamente. }\end{array}$ & 4,7 & 4,4 & 4,0 & 5,2 & 3,0 & 2,2 & 3,5 & 3,6 \\
\hline
\end{tabular}

Nota: Valores em negrito não foram estatisticamente significantes na comparação entre os anos pelo teste qui quadrado de Pearson ajustado, ao nível de $5 \%$.

Fonte: PNS (2013 e 2019).

\section{Discussão}

Este estudo investigou a prevalência da HAS e os indicadores de cuidados em saúde entre os hipertensos, com 18 anos ou mais. Utilizamos a medida da HAS autorreferida que, embora possa apresentar alguma limitação devido à seletividade, é considerada uma boa medida em estudos populacionais ${ }^{21}$. Os resultados das razões de prevalência ajustados por características sociodemográficas para os anos de 2008-2013-2019 demonstraram que a prevalência é maior entre as mulheres, nas pessoas mais velhas, com baixa escolaridade e residentes das regiões Sul e Sudeste, como reportado por estudos anteriores ${ }^{22,23}$. Com relação aos cuidados em saúde pela população hipertensa, os indicadores apresentaram bons resultados com destaque para a cobertura de assistência médica nos últimos 12 meses e a proporção elevada de usuários que relataram o uso da medicação nas duas últimas semanas, acima de $80 \%$ nos dois anos.

A busca por cuidados de saúde representa, em partes, aspectos de necessidade, mas fatores re- lacionados à oferta contribuem para facilitar ou dificultar o acesso. A expansão da ESF a partir dos anos 2000 representou uma nova estratégia para a atenção primária no país, centrada na família e na comunidade, considerada um dos grandes avanços do SUS por seu papel fundamental na ampliação do acesso aos serviços de saúde e na melhoria de indicadores de atenção à saúde ${ }^{24}$. Com destaque para o trabalho das equipes de saúde da família que, através de ações educativas de promoção à saúde, orientação quanto à utilização dos serviços médicos, além de outras estratégias que visam a vigilância e promoção da saúde, contribuem para os bons resultados alcançados ${ }^{25}$. Em 2008, 47,7\% dos domicílios no Brasil estavam cadastrados pela ESF. Em 2019, esse percentual foi de 60,0\%, representando um aumento de 6,4 pontos percentuais em relação à 2013. A cobertura populacional da ESF passou de 50,9\% em 2008, para 56,1\% em 2013 e $62,6 \%$ em 2019. Dentre os domicílios cadastrados há pelo menos um ano, a proporção de visita mensal de membros da equipe de saúde da família nos domicílios foi de 47,2\% em 2013 e de $38,4 \%$ em 2019 (resultados não mostrados). 
Tabela 4. Indicadores de cuidados em saúde pelos indivíduos com hipertensão arterial sistêmica segundo faixa etária.

\begin{tabular}{|c|c|c|c|c|c|c|c|c|c|c|c|c|}
\hline \multirow[b]{2}{*}{ Variáveis } & \multicolumn{6}{|c|}{2013} & \multicolumn{6}{|c|}{2019} \\
\hline & $\begin{array}{c}\text { Total } \\
(\%)\end{array}$ & $\begin{array}{c}18-29 \\
(\%)\end{array}$ & $\begin{array}{c}30-59 \\
(\%)\end{array}$ & $\begin{array}{c}\text { 60-64 } \\
(\%)\end{array}$ & $\begin{array}{c}65-74 \\
(\%)\end{array}$ & $\begin{array}{c}75 \text { ou } \\
\text { mais } \\
(\%)\end{array}$ & $\begin{array}{c}\text { Total } \\
(\%)\end{array}$ & $\begin{array}{c}18-29 \\
(\%)\end{array}$ & $\begin{array}{c}30-59 \\
(\%)\end{array}$ & $\begin{array}{c}\text { 60-64 } \\
(\%)\end{array}$ & $\begin{array}{c}65-74 \\
(\%)\end{array}$ & $\begin{array}{c}75 \text { ou } \\
\text { mais } \\
(\%)\end{array}$ \\
\hline $\begin{array}{l}\text { Tomou medicamentos } \\
\text { para hipertensão nas } \\
\text { últimas duas semanas }\end{array}$ & 81,6 & 35,4 & 77,2 & 88,7 & 91,5 & 92,3 & 88,0 & 40,3 & 83,2 & 93,2 & 95,2 & 96,7 \\
\hline $\begin{array}{l}\text { Recebeu assistência nos } \\
\text { últimos } 12 \text { meses para } \\
\text { hipertensão }\end{array}$ & 69,7 & 60,6 & 68,3 & 72,1 & 73,0 & 71,1 & 72,2 & 62,9 & 71,1 & 74,3 & 73,6 & 73,1 \\
\hline Local do atendimento & & & & & & & & & & & & \\
\hline Unidade Básica de Saúde & 46,0 & 36,1 & 46,8 & 45,6 & 47,4 & 43,0 & 45,8 & 47,1 & 45,5 & 48,2 & 47,8 & 41,3 \\
\hline Consultório particular & 24,5 & 17,5 & 23,9 & 25,5 & 24,2 & 29,3 & 28,8 & 17,6 & 28,0 & 28,3 & 29,0 & 33,6 \\
\hline Atendimento pelo SUS & 65,6 & 67,0 & 66,2 & 65,7 & 66,4 & 61,1 & 65,6 & 77,7 & 66,1 & 67,3 & 66,0 & 59,7 \\
\hline $\begin{array}{l}\text { Última consulta com } \\
\text { mesmo médico das } \\
\text { consultas anteriores }\end{array}$ & 56,5 & 39,8 & 55,0 & 55,2 & 60,1 & 63,0 & 51,9 & 37,6 & 49,6 & 53,4 & 55,1 & 56,1 \\
\hline $\begin{array}{l}\text { Fez todos os exames } \\
\text { solicitados }\end{array}$ & 91,9 & 88,5 & 89,9 & 91,7 & 95,2 & 96,3 & 89,6 & 86,9 & 88,0 & 91,2 & 90,2 & 92,6 \\
\hline $\begin{array}{l}\text { Pactuação de plano } \\
\text { de cuidados com } \\
\text { profissionais da saúde }\end{array}$ & 96,5 & 94,1 & 96,7 & 97,7 & 96,9 & 94,7 & 95,1 & 93,4 & 95,3 & 95,7 & 95,2 & 94,0 \\
\hline $\begin{array}{l}\text { Fez todas as consultas } \\
\text { ou foi encaminhado e } \\
\text { conseguiu fazer consultas } \\
\text { com especialistas }\end{array}$ & 87,3 & 67,0 & 84,6 & 92,8 & 93,0 & 89,8 & 90,4 & 77,9 & 88,4 & 92,0 & 92,6 & 93,3 \\
\hline $\begin{array}{l}\text { Alguma vez esteve } \\
\text { internado por causa } \\
\text { da hipertensão ou } \\
\text { complicação }\end{array}$ & 14,0 & 6,9 & 12,6 & 15,3 & 15,9 & 17,9 & 12,7 & 10,7 & 11,9 & 14,1 & 12,4 & 14,6 \\
\hline $\begin{array}{l}\text { Tem suas atividades } \\
\text { habituais limitadas } \\
\text { devido à hipertensão de } \\
\text { forma intensa ou muito } \\
\text { intensamente. }\end{array}$ & 4,7 & 2,1 & 4,7 & 4,2 & 4,2 & 6,4 & 3,0 & 2,9 & 3,1 & 2,9 & 2,6 & 3,3 \\
\hline
\end{tabular}

Fonte: PNS (2013 e 2019).

A despeito dos bons resultados apresentados, ainda são muitos os desafios relativos à melhoria do controle e tratamento da HAS no país. Em um estudo incluindo 44 países de baixa e média renda, o Brasil apresentou bom desempenho no manejo da HAS comparado aos demais países, mas os autores não descartam a possibilidade de um subdiagnóstico elevado ${ }^{26}$. Considerando diferentes mensurações da HAS, Malta et al. ${ }^{27}$ reportam as seguintes prevalências entre os brasileiros em 2013: $21,4 \%$ pelo critério autorreferido, $22,8 \%$ pela aferição da pressão arterial e 32,3\% através da pressão aferida e/ou relato de uso de medicação.
As mulheres continuam apresentando maior prevalência de HAS em relação aos homens, reforçando o "paradoxo da saúde-sobrevivência na relação homem-mulher”. Termo designado na literatura de diferencial de gênero em saúde para ressaltar o fato de que, embora vivam mais do que os homens, as mulheres apresentam mais morbidades e buscam mais por serviços de saúde. Possíveis explicações para a aparente contradição envolvem fatores biológicos, diferencial na exposição aos fatores de riscos, fatores psicológicos e socioeconômicos ${ }^{28}$. Ao mesmo tempo, ao procurarem os serviços de saúde com mais frequência, 
Tabela 5. Indicadores de cuidados em saúde pelos indivíduos com hipertensão arterial sistêmica e cobertura populacional da ESF de saúde por região.

\begin{tabular}{|c|c|c|c|c|c|c|c|c|c|c|c|c|}
\hline \multirow[b]{2}{*}{ Variáveis } & \multicolumn{6}{|c|}{2013} & \multicolumn{6}{|c|}{2018} \\
\hline & $\begin{array}{c}\text { Total } \\
(\%)\end{array}$ & Norte & Nordeste & Sudeste & Sul & $\begin{array}{c}\text { Centro- } \\
\text { Oeste }\end{array}$ & $\begin{array}{c}\text { Total } \\
(\%)\end{array}$ & Norte & Nordeste & Sudeste & Sul & $\begin{array}{c}\text { Centro- } \\
\text { Oeste }\end{array}$ \\
\hline $\begin{array}{l}\text { Tomou medicamentos } \\
\text { para hipertensão nas } \\
\text { últimas duas semanas }\end{array}$ & 81,6 & 72,0 & 78,4 & 83,8 & 83,8 & 79,8 & 88,0 & 81,9 & 87,5 & 88,8 & 89,6 & 86,2 \\
\hline $\begin{array}{l}\text { Recebeu assistência nos } \\
\text { últimos } 12 \text { meses para } \\
\text { hipertensão }\end{array}$ & 69,7 & 71,9 & 67,9 & 71,6 & 68,4 & 64,9 & 72,2 & 74,7 & 71,9 & 73,0 & 70,8 & 68,5 \\
\hline \multicolumn{13}{|l|}{ Local do atendimento } \\
\hline Unidade Básica de Saúde & 46,0 & 49,8 & 45,2 & 45,0 & 48,6 & 46,8 & 45,8 & 50,6 & 48,4 & 41,5 & 53,2 & 46,1 \\
\hline Consultório particular & 24,5 & 17,9 & 18,8 & 27,7 & 25,1 & 25,9 & 28,8 & 20,9 & 21,2 & 34,0 & 28,2 & 29,4 \\
\hline Atendimento pelo SUS & 65,6 & 70,5 & 73,7 & 61,5 & 64,9 & 64,4 & 65,6 & 72,2 & 74,2 & 60,1 & 66,5 & 64,0 \\
\hline $\begin{array}{l}\text { Última consulta com } \\
\text { mesmo médico das } \\
\text { consultas anteriores }\end{array}$ & 56,5 & 44,2 & 46,7 & 61,5 & 59,8 & 56,2 & 51,9 & 42,6 & 47,2 & 54,9 & 55,9 & 48,1 \\
\hline $\begin{array}{l}\text { Fez todos os exames } \\
\text { solicitados }\end{array}$ & 91,9 & 88,7 & 87,1 & 93,7 & 93,9 & 92,5 & 89,6 & 87,8 & 86,9 & 90,0 & 92,6 & 91,3 \\
\hline $\begin{array}{l}\text { Pactuação de plano } \\
\text { de cuidados com } \\
\text { profissionais da saúde }\end{array}$ & 96,5 & 95,6 & 96,1 & 96,7 & 96,9 & 96,6 & 95,1 & 96,2 & 95,3 & 94,9 & 94,7 & 95,3 \\
\hline $\begin{array}{l}\text { Fez todas as consultas } \\
\text { ou foi encaminhado e } \\
\text { conseguiu fazer consultas } \\
\text { com especialistas }\end{array}$ & 87,3 & 88,2 & 81,6 & 88,3 & 91,9 & 86,6 & 90,4 & 86,4 & 87,4 & 91,8 & 90,3 & 91,2 \\
\hline $\begin{array}{l}\text { Alguma vez esteve } \\
\text { internado por causa } \\
\text { da hipertensão ou } \\
\text { complicação }\end{array}$ & 14,0 & 14,9 & 16,1 & 12,7 & 14,5 & 13,3 & 12,7 & 14,4 & 13,8 & 11,5 & 12,8 & 15,0 \\
\hline $\begin{array}{l}\text { Tem suas atividades } \\
\text { habituais limitadas } \\
\text { devido à hipertensão de } \\
\text { forma intensa ou muito } \\
\text { intensamente. }\end{array}$ & 4,7 & 5,0 & 5,5 & 4,1 & 5,0 & 4,6 & 3,0 & 4,1 & 3,6 & 2,6 & 3,2 & 2,8 \\
\hline $\begin{array}{l}\text { \% de moradores em } \\
\text { domicílios cadastrados } \\
\text { na ESF }\end{array}$ & 56,1 & 53,4 & 68,1 & 48,2 & 58,5 & 54,5 & 62,6 & 62,2 & 73,3 & 54,6 & 67,6 & 60,1 \\
\hline
\end{tabular}

Nota: Valores em negrito não foram estatisticamente significantes na comparação entre os anos pelo teste qui quadrado de Pearson ajustado, ao nível de 5\%.

Fonte: PNS (2013 e 2019).

as mulheres têm maiores chances de obter o diagnóstico clínico da condição.

Por outro lado, estudos que utilizam a aferição da pressão arterial ao invés do diagnóstico clínico reportam maior diagnóstico entre os homens e os grupos de status socioeconômico mais baixo ${ }^{29}$, o que pode sugerir a presença de subdiagnóstico para essa população. Um indício disso é que a principal queixa entre homens se relaciona com a flexibilização de horários e o tempo disponível para a procura dos cuidados médi- $\cos ^{30}$. O caráter assintomático da doença também pode fazer com que indivíduos mais jovens que tenham maior dificuldade ou que não sintam a necessidade de procurar serviços médicos não obtenham o diagnóstico.

Os resultados também apontam para a persistência de desigualdades regionais. A alta prevalência no Sul e Sudeste ao longo dos anos pode refletir tantos fatores demográficos, já que essas regiões apresentam maior proporção de idosos ${ }^{27}$, como iniquidades regionais quanto à estrutura 
e oferta dos serviços, uma vez que os residentes do Sul e Sudeste apresentam maior acesso aos serviços de saúde comparados aos residentes das demais regiões ${ }^{31}$.

\section{Conclusão}

As respostas contínuas e integradas em redes continuam sendo um desafio para os sistemas de saúde. Não um problema restrito ao Brasil, mas observado também em outros países, inclusive nos desenvolvidos ${ }^{32}$. Evidências recentes revelam deficiências na longitudinalidade e na integralidade do cuidado para os usuários com $\mathrm{HAS}^{33}$. O fortalecimento dos vínculos entre profissionais de saúde e usuários, a fixação e a formação dos profissionais de saúde, bem como o compartilhamento de informação entre os profissionais por meio da adoção de prontuários eletrônicos e de mecanismos de referência e contrarreferência bem estabelecidos são alguns desafios enfrentados na atenção primária. Além das dificuldades encontradas no cuidado especializado e nos serviços de diagnose ${ }^{34}$.

Destacamos ainda a necessidade de ações voltadas à conscientização da doença e dos riscos envolvidos junto aos usuários, reforçando a importância do tratamento medicamentoso continuado e da adoção de estilos de vida saudáveis. A implementação dos programas Hiperdia e Farmácia Popular do Brasil foram sem dúvida iniciativas importantes na ampliação do acesso aos medicamentos. Todavia, a ausência de informação sobre o uso correto da medicação, bem como a relativização dos riscos pelos usuários devido à ausência de sintomas e controle da pressão ar- terial, acarreta desde falhas no uso ao abandono completo do tratamento. A complexidade do regime da medicação, o vínculo entre profissionais e usuários e fatores relacionados à demanda $\mathrm{e}$ à oferta também são preditores importantes da aderência ${ }^{9,10,13,14}$.

A elevada prevalência de fatores de risco no estilo de vida da população brasileira também merece atenção ${ }^{35}$. O abismo entre a pactuação de planos de cuidado e a modificação efetiva dos estilos de vida prejudiciais por parte dos indivíduos dificulta a prevenção e aumenta os riscos de complicações por HAS. Entretanto, a responsabilidade quanto às mudanças no estilo de vida não deve ser creditada somente ao indivíduo, sendo a responsabilidade do Estado parte subjacente ao processo de mudança proporcionando, por exemplo, lugares públicos adequados para a prática de atividades físicas e desenvolvendo políticas públicas adequadas para uma alimentação saudável.

Por fim, um agravante para o sistema de saúde brasileiro é o contexto de crise econômica e um quadro fiscal deteriorado. Nos anos 2000, como continuidade de uma política iniciada em meados dos anos 1990, observamos importante orientação política voltada à priorização da atenção primária, com a criação de sucessivos incentivos e crescimento da execução orçamentária à atenção básica ${ }^{36}$. A partir de 2015, notamos uma inflexão da política econômica com governos estaduais e municipais adotando uma agenda de austeridade fiscal como resposta à crise econômica, com consequências diretas sobre os gastos sociais e de saúde. Os efeitos deletérios dessa política sobre os resultados em saúde podem se traduzir em múltiplas dimensões na esfera individual e social ${ }^{37}$.

\section{Colaboradores}

Todas as autoras participaram igualmente da concepção e desenho da pesquisa, análise estatística e interpretação dos dados, redação do manuscrito, revisão crítica do manuscrito quanto ao conteúdo intelectual importante até a aprovação da versão final. 


\section{Referências}

1. Ettehad D, Emdin CA, Kiran A, Anderson SG, Callender T, Emberson J, Chalmers J, Rodgers A, Rahimi K. Blood pressure lowering for prevention of cardiovascular disease and death: A systematic review and meta -analysis. Lancet 2016; 387(10022):957-967.

2. Yang J, Zheng Y, Gou X, Pu K, Chen Z, Guo Q, Ji R, Wang H, Wang Y, Zhou Y. Prevalence of comorbidities and its effects in patients infected with SARS-CoV-2. Int J Infect Dis 2020; 94(April):91-95.

3. Abate S, Checkol Y, Mantedafro B, Basu B. Prevalence and risk factors of mortality among hospitalized patients with COVID-19: A systematic review and Meta-analysis. Bull World Heal Organ 2020; 419(April):1-26.

4. Nilson EAF, Andrade RCS, Brito DA, Michele Lessa O. Custos atribuíveis a obesidade, hipertensão e diabetes no Sistema Único de Saúde, Brasil, 2018. Rev Panam Salud Publica 2020; 44:e32..

5. Campolina AG, Adami F, Santos JLF, Lebrão ML. A transição de saúde e as mudanças na expectativa de vida saudável da população idosa: possíveis impactos da prevenção de doenças crônicas. Cad Saude Publica 2013; 29(6):1217-1229.

6. Mills KT, Stefanescu A, He J. The global epidemiology of hypertension. Nat Rev Nephrol 2020; 16(4):223237.

7. Colhoun HM, Hemingway H, Poulter NR. Socio-economic status and blood pressure: An overview analysis. J Hum Hypertens 1998;12:91-110.

8. Macinko J, Mendonça CS. Estratégia Saúde da Família, um forte modelo de Atenção Primária à Saúde que traz resultados. Saude Debate 2018; 42:18-37.

9. Paula PAB, Stephan-Souza AI, Vieira RCPA, Alves TNP. O uso do medicamento na percepção do usuário do Programa Hiperdia The use of medication in the perception of users Hiperdia Program. Cien Saude Colet 2011; 16(5):2623-2633.

10. Carvalho ALM, Leopoldino RWD, Silva JEG, Cunha CP. Adesão ao tratamento medicamentoso em usuários cadastrados no Programa Hiperdia no município de Teresina (PI). Cien Saude Colet 2012;17(7):18851892.

11. Costa KS, Tavares NUL, Mengue SS, Pereira MA, Malta DC, Silva Júnior JB. Obtenção de medicamentos para hipertensão e diabetes no Programa Farmácia Popular do Brasil: resultados da Pesquisa Nacional de Saúde, 2013. Epidemiol Serv Saude 2016; 25(1):33-44.

12. Sabaté E. Adherence to long-term therapies: evidence for action. Geneva: World Health Organization; 2003.

13. Duarte MTC, Cyrino AP, Cerqueira ATAR, Nemes MIB, Iyda M. Motivos do abandono do seguimento médico no cuidado a portadores de hipertensão arterial: a perspectiva do sujeito. Cien Saude Colet 2010; 15(5):2.603-2.610.

14. Gewehr DM, Bandeira VAC, Gelatti GT, Colet CF, Oliveira KR. Adesão ao tratamento farmacológico da hipertensão arterial na Atenção Primária à Saúde. Saude Debate 2018; 42(116):179-190.

15. Malta DC, Stopa SR, Andrade SSCA, Szwarcwald CL, Silva Júnior JB, Reis AAC. Cuidado em saúde em adultos com hipertensão arterial autorreferida no Brasil segundo dados da pesquisa nacional de saúde, 2013. Rev Bras Epidemiol 2015; 18:109-122.
16. Silva PLN, Pessoa DGC, Lila MF. Análise estatística de dados da PNAD: incorporando a estrutura do plano amostral - Statistical analysis of data from PNAD: incorporating the sample design. Cien Saude Colet 2002; 7(4):659-670.

17. Stopa SR, Szwarcwald CL, Oliveira MM, Gouvea ECDP, Vieira MLFP, Freitas MPS, Sardinha LMV, Macário EM. Pesquisa Nacional de Saúde 2019: histórico, métodos e perspectivas. Epidemiol Serv Saude 2020; 29(5):e2020315.

18. Souza-Júnior PRB, Freitas MPS, Antonaci GA, Szwarcwald CL. Desenho da amostra da Pesquisa Nacional de Saúde 2013. Epidemiol Serv Saude 2015; 24(2):207216.

19. Coutinho LMS, Scazufca M, Menezes PR. Métodos para estimar razão de prevalência em estudos de corte transversal. Rev Saude Publica 2008; 42(6):992-998.

20. Lobo LAC, Canuto R, Dias-da-Costa JS, Pattussi MP. Tendência temporal da prevalência de hipertensão arterial sistêmica no Brasil. Cad Saude Publica 2017; $33(6)$.

21. Lima-Costa MF, Peixoto SV, Firmo JOA. Validade da hipertensão arterial auto-referida e seus determinantes (projeto Bambuí). Rev Saude Publica 2004;38(5):637-642.

22. Andrade SSA, Stopa SR, Brito AS, Chueri PS, Szwarcwald CL, Malta DC. Prevalência de hipertensão arterial autorreferida na população brasileira: análise da Pesquisa Nacional de Saúde, 2013. Epidemiol Serv Saude 2015; 24(2):297-304.

23. Muniz LC, Cascaes AM, Wehrmeister FC, MartínezMesa J, Barros AJD, Menezes AMB. Tendência temporal de hipertensão arterial autorreferida em adultos: Uma análise da Pesquisa Nacional por Amostra de Domicílios, 1998-2008. Cad Saude Publica 2012; 28(8):1599-1607.

24. Alexandre LBSP. Os avanços e desafios do SUS nas duas décadas de existência. In: Martinari, organizador. SUS: Sistema Único de Saúde - antecedentes, percurso, perspectivas e desafios. $2^{\circ}$ ed. São Paulo; 2015. p. 149-166.

25. Rodrigues CRF. Do Programa de Saúde da Família à Estratégia Saúde da Família. In: Martinari, organizador. SUS: Sistema Único de Saúde - antecedentes, percurso, perspectivas e desafios. $2^{\circ}$ ed São Paulo; 2015. p. 131-148.

26. Geldsetzer P, Manne-Goehler J, Marcus ME, Ebert C, Zhumadilov Z, Wesseh CS, Tsabedze L, Supiyev A, Sturua L, Bahendeka SK, Sibai AM, Quesnel-Crooks S, Norov B, Mwangi KJ, Mwalim O, Wong-McClure R, Mayige MT, Martins JS, Lunet N, Labadarios D, Karki KB, Kagaruki GB, Jorgensen JMA, Hwalla NC, Houinato D, Houehanou C, Msaidié M, Guwatudde D, Gurung MS, Gathecha G, Dorobantu M, Damasceno A, Bovet P, Bicaba BW, Aryal KK, Andall-Brereton G, Agoudavi K, Stokes A, Davies JI, Bärnighausen T, Atun R, Vollmer S, Jaacks LM.. The state of hypertension care in 44 low-income and middle-income countries: a cross-sectional study of nationally representative individual-level data from 1.1 million adults. Lancet 2019; 394(10199):652-662. 
27. Malta DC, Gonçalves RPF, Machado ÍE, Freitas MIF, Azeredo C, Szwarcwald CL. Prevalência da hipertensão arterial segundo diferentes critérios diagnósticos, Pesquisa Nacional de Saúde. Rev Bras epidemiol 2018; 21:e180021.

28. Pinheiro RS, Viacava F, Travassos C, Brito AS. Gênero, morbidade, acesso e utilização de serviços de saúde no Brasil. Cien Saude Colet 2002; 7(4):687-707.

29. Alves RFS, Faerstein E. Educational inequalities in hypertension: Complex patterns in intersections with gender and race in Brazil. Int J Equity Health 2016; 15(1):1-9.

30. Gomes R, Nascimento EF, Araújo FC. Por que os homens buscam menos os serviços de saúde do que as mulheres? As explicações de homens com baixa escolaridade e homens com ensino superior. Cad Saude Publica 2007; 23(3):565-574.

31. Stopa SR, Malta DC, Monteiro CN, Szwarcwald CL, Goldbaum M, Cesar CLG. Acesso e uso de serviços de saúde pela população brasileira, Pesquisa Nacional de Saúde 2013. Rev Saude Publica 2017; 51.

32. Mendes EV. Entrevista: A abordagem das condições crônicas pelo Sistema Único de Saúde. Cien Saude Colet 2018; 23(2):431-435.

33. Andrade MV, Noronha K, Oliveira CDL, Cardoso CS, Calazans JA, Julião NA, Souza A, Tavares PA. Análise da linha de cuidado para pacientes com diabetes mellitus e hipertensão arterial: a experiência de um município de pequeno porte no Brasil. Rev Bras Estud Popul 2019; 36.

34. Giovanella L, Mendonça MHMM. Atenção Primária à Saúde. In: Giovanella L. Políticas e sistema de saúde no Brasil. Rio de Janeiro: Editora Fiocruz; 2014. p. 493-546.

35. Malta DC, Andrade SSCA, Stopa SR, Pereira CA, Szwarcwald CL, Silva Júnior JB, Reis AAC. Estilos de vida da população brasileira: resultados da Pesquisa Nacional de Saúde, 2013. Epidemiol Serv Saude 2015; 24(2):217-226.

36. Castro ALB, Machado CV. A política de atenção primária à saúde no Brasil: notas sobre a regulação $\mathrm{e}$ o financiamento federal. Cad Saude Publica 2010; 26(4):693-705.

37. Paes-Sousa R, De Andrade Schramm JM, Pereira Mendes LV. Fiscal Austerity and the health sector: The cost of adjustments. Cien Saude Colet 2019; 24(12):4375-4384.

Artigo apresentado em 29/01/2021

Aprovado em 20/04/2021

Versão final apresentada em 22/04/2021

Editores-chefes: Maria Cecília de Souza Minayo, Romeu Gomes, Antônio Augusto Moura da Silva 
